\title{
Possible Involvement of Iron-Dependent Lipid Peroxidation in the Delayed Neuronal Death of Rat Brain after a Transient Ischemia
}

\author{
Hiroyuki AraI, ${ }^{1}$ Koji MachII, ${ }^{1}$ Takehide Tsuda, ${ }^{1}$ \\ Kyuya Kogure, ${ }^{1}$ Takao WatAnABE, ${ }^{2}$ and Minoru NAKAnO ${ }^{3}$,* \\ ${ }^{1}$ Department of Neurology, Institute of Brain Diseases and \\ ${ }^{2}$ Department of Environmental Health, \\ Tohoku University School of Medicine, Sendai 980, Japan \\ ${ }^{3}$ College of Medical Care and Technology, \\ Gunma University, Maebashi 371, Japan
}

(Received June 30, 1987)

\begin{abstract}
Summary Lipid peroxide levels in rat hippocampus and cortex were determined at 6,24 , and $48 \mathrm{~h}$ following ischemia for a period of $20 \mathrm{~min}$. A significant $(p<0.05)$ but transient increase in lipid peroxide formation was found in the hippocampus at $24 \mathrm{~h}$ following ischemia. Regional iron concentration determined by inductively coupled plasma (ICP) emission spectrometry revealed that the concentration of iron in the hippocampus was also significantly elevated at $24 \mathrm{~h}$ following ischemia, suggesting that in the hippocampus the increase in the concentration of iron resulted in promotion of lipid peroxidation. Though lipid peroxide and iron levels were reversed at $48 \mathrm{~h}$ following ischemia, iron-dependent lipid peroxidation was possibly involved in the onset of delayed neuronal death in the rat hippocampus.
\end{abstract}

Key Words: ischemia, delayed neuronal death, hippocampus, lipid peroxidation, iron

Delayed neuronal death in pyramidal cells of CA 1 subfield of the hippocampus following brief forebrain ischemia has received considerable attention in recent years [1-3]. Delayed neuronal death is characterized by the late onset of neuronal damage in selective vulnerable regions after a restoration of blood flow. Kirino et al. [4] observed by light microscopy that in the CA 1 subfield most of the pyramidal neurons did not show overt morphological changes up to 1 day following $20 \mathrm{~min}$ of ischemia, but that neuronal destruction became dramatically pronounced at $2-4$ days.

*To whom correspondence should be addressed. 
The biochemical mechanisms underlying the delayed onset of neuronal injury are not yet known, although histopathological alterations have been well documented. Measurement of regional cerebral blood flow and glucose utilization in the rat model of ischemia up to $30 \mathrm{~min}$ indicates that these parameters did not explain the delayed onset and slow progression of destruction of susceptible neurons $[5,6]$. Measurement of energy reserves in the CA 1 subfield of the hippocampus in the gerbil model of ischemia demonstrates no decrease in ATP during the period of neuronal deterioration [7]. These findings clearly indicate that delayed neuronal death is not due to failure of the energy-producing system in mitochondria. Another attractive hypothesis is that excessive influx of $\mathrm{Ca}^{2+}$ into the neurons following ischemia triggers deleterious reactions, resulting in neuronal death [8]. Although massive accumulation of $\mathrm{Ca}^{2+}$ in cytoplasma of injured neurons was demonstrated [9], an important question still remains whether the observed increase in cytosolic $\mathrm{Ca}^{2+}$ concentration proceeds the neuronal damage or is only its consequence.

On the other hand, membrane lipid peroxidation has been implicated as one of the potential mechanisms leading to postischemic brain injury [10, 11]. In our previous study, we demonstrated that brain lipid peroxidation is catalyzed by endogenous iron in the presence of oxygen [12]. Thus, if the brain iron level was elevated during and/or following ischemia, in vivo lipid peroxidation would be expected to occur. To our knowledge no report has ever appeared, concerning on postischemic iron metabolism. The purpose of the present study was to clarify whether or not lipid peroxidation as monitored by thiobarbituric acid-reactive substance formation is responsible for delayed neuronal death in the rat hippocampus, with special reference to brain iron metabolism.

\section{MATERIALS AND METHODS}

Operative procedures. Male Wistar rats weighing 220-280 g were purchased from Japan Clea Laboratory. The animals were allowed free access to commercial diet and tap water until experiments were started, at which time the rats were subjected to four-vessel occlusion as described by Pulsinelli and Brierley [13]. On the day following electrocautery of vertebral arteries, bilateral carotid arteries were clipped to produce four-vessel occlusion for $20 \mathrm{~min}$. The four-vessel-occluded rats became unresponsive and lost their righting reflex after bilateral carotid artery clipping. The animals which did not lose their righting reflex or showed seizure-like movement during the ischemia were excluded from further study. The rats were maintained for 6,24 or $48 \mathrm{~h}$ following ischemia and then killed under anesthesia with pentobarbital by transcardiac perfusion with $0.9 \% \mathrm{NaCl}$ for $2 \mathrm{~min}$. The $0.9 \% \mathrm{NaCl}$ solution was prepared with chelex-100-treated redistilled water just perior to perfusion. The brains were removed and rapidly placed on an ice-cold glass stage on which they were dissected according to the method of Glowinski and Iversen [14]. 
Analytical procedures. For the determination of lipid peroxides, rat cortex and hippocampus were homogenized in ice-cold $0.1 \mathrm{M}$ Tris- $\mathrm{HCl}$ buffer ( $\mathrm{pH} 7.4)$. The buffer solution was previously deoxygenated with $100 \% \mathrm{~N}_{2}$ at least $30 \mathrm{~min}$ before homogenization. Lipid peroxide was measured as thiobarbituric acid-reactive substances (TBARS) according to the method described by Ohkawa et al. [15]. The protein concentration was determined by the method of Lowry et al. [16].

For the analysis of iron, dissected brains were carefully placed in individual glass containers and then dried overnight at $110^{\circ} \mathrm{C}$ and weighed. The samples were wet digested with $1: 1(\mathrm{v} / \mathrm{v}) \mathrm{HNO}_{3}: \mathrm{HClO}_{4}(60 \%)$. The digests were analyzed by inductively coupled plasma (ICP) emission spectrometry as described by Ikeda et al. [17]. The concentration of iron was expressed as $\mu \mathrm{g}$ per dry weight basis. All glassware employed was soaked in 10\% nitric acid at least for $24 \mathrm{~h}$ and rinsed at least 3 times with chelex-100-treated redistilled water. Stainless steel equipments were coated with Teflon. These precautions were taken to prevent possible contamination of metal ions.

\section{RESULTS}

As shown in Fig. 1, normal rat hippocampus and cortex obtained from brains of sham-operated animals (vertebral arteries electrocauterized only) contained $1.47 \pm 0.03$ ( $n=6$, hippocampus) and $1.62 \pm 0.16(n=5$, cortex) nmol of TBARS per $\mathrm{mg}$ protein. These values were found not to be significantly different. In the hippocampus, the TBARS value at $24 \mathrm{~h}$ following ischemia was significantly higher than that of sham-operated rats $(p<0.05)$, but not significant at $48 \mathrm{~h}$ following ischemia. In the cortex, however, a significant decrease in TBARS formation was found at $6 \mathrm{~h}$ following ischemia $(p<0.05)$.

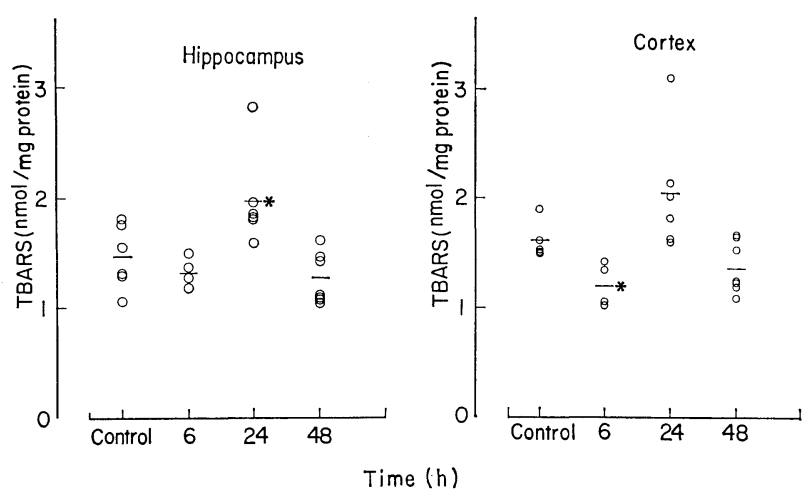

Fig. 1. Lipid peroxide levels of rat hippocampus (left) and cortex (right) at 6, 24, and 48 $\mathrm{h}$ following ischemia for $20 \mathrm{~min}$. Each horizontal line indicates the mean value of 4-7 animals. Control, Sham-operated group (vertebral arteries electro-cauterized only). * Significant different $(p<0.05)$ compared with control group.

Vol. 3, No. 3, 1987 
Table 1. Changes in regional concentration of iron following ischemia for $20 \mathrm{~min}$.

\begin{tabular}{|c|c|c|c|c|}
\hline \multirow{2}{*}{ Brain region } & \multirow{2}{*}{ Control } & \multicolumn{3}{|c|}{ Time after ischemia (h) } \\
\hline & & 6 & 24 & 48 \\
\hline Hippocampus & $7.61 \pm 0.77$ & $7.93 \pm 0.42$ & $9.69 \pm \frac{ \pm 1.49 *}{(4)}$ & $8.79 \pm 1.78$ \\
\hline Cortex & $11.56 \pm 1.51$ & $9.26 \pm 0.52 *$ & $8.63 \pm 0.69 *$ & $8.77 \pm 0.69$ \\
\hline Thalamus & $14.80 \pm 7.09$ & $9.69 \pm 1.34$ & $11.88 \pm \frac{ \pm 2.74}{(4)}$ & $9.56 \pm 1.60$ \\
\hline Hypothalamus & $14.17 \frac{ \pm 3.31}{(3)}$ & $11.90 \pm \frac{ \pm}{(4)} .51$ & $14.34 \pm \frac{ \pm}{(4)} .30$ & $12.45 \frac{ \pm 1.53}{(5)}$ \\
\hline Striatum & $13.47 \pm \frac{ \pm 1.90}{(3)}$ & $12.70 \pm \frac{ \pm}{(4)} .03$ & $11.61 \pm \frac{ \pm 1.08}{(3)}$ & $10.99 \frac{ \pm 1.60}{(4)}$ \\
\hline
\end{tabular}

Each value represents mean $\pm \mathrm{SD}(\mu \mathrm{g} / \mathrm{g}$ dry weight). Numbers of animals in parentheses.

* Significant difference $(p<0.05)$ compared with control group.

Table 1 shows the regional changes in the concentration of iron following ischemia. In normal rat brain obtained in the same fashion, the concentration of iron was lowest in the hippocampus and the cortex contained about 1.5 fold of iron over the hippocampus. In the hippocampus a significant increase in the concentration of iron was found at $24 \mathrm{~h}$ following ischemia $(p<0.05)$, but not at 48 $\mathrm{h}$ following ischemia. In the cortex, the concentration of iron during the postischemic period up to 2 days was found to be significantly lower than that of shamoperated rats $(p<0.05)$. Thalamus, hypothalamus, and striatum contained a relatively high amount of iron, which showed neither increase nor decrease during the 2 day period following ischemia.

\section{DISCUSSION}

As shown in Table 1, brain iron occurs in different concentrations in different brain regions. Furthermore, postischemic iron metabolism is also different regionally. The most characteristic feature of postischemic iron metabolism is that in the hippocampus a transient increase in the concentration of iron was found at $24 \mathrm{~h}$ following ischemia. Iron-positive red blood cells were not histochemically stained by the Perls' + DAB (diaminobenzidine) reaction after rat brains had been perfused with $10 \%$ formalin in the same fashion as the present experiments (data not shown), indicating that parenchymal iron was measured, not that of contaminating with blood. Regional values of parenchymal iron in normal rat brain determined by ICP emission spectrometry are, on the whole, consistent with the histochemical picture of iron reported by Hill and Switzer [18].

Intracellular iron is mainly in the form of ferritin which is considered not to promote lipid peroxidation. The remaining iron is included in low-molecularweight iron complexes as well as that found in enzymes and myelin sheets [19]. One such low-molecular-weight iron complex, iron-nucleotide chelate, is considered to be a powerful initiator of lipid peroxidation [20]. The concentration 
of such a low-molecular-weight iron should be extremely low as compared with that of ferritin. Zaleska and Floyd stressed the importance of endogenous iron in lipid peroxidation of rat brain by demonstrating a linear relationship between iron content of brain regions and their ability to produce lipid peroxide [21]. We also demonstrated that brain lipid peroxidation was completely inhibited in the presence of deferroxamine, a potent iron chelator [12]. Therefore, it seems reasonable to consider that in the hippocampus the increase in the concentration of iron seen at $24 \mathrm{~h}$ following ischemia resulted in promotion of lipid peroxidation at the same time. Isotope study is further required to demonstrate the incorporation and accumulation of iron in the hippocampus during postischemic period.

Kirino et al. have recently reported that electron microscopic changes were already found in the neurons of CA 1 subfield of hippocampus at $24 \mathrm{~h}$ following ischemia, although these neurons still appeared intact by light microscopy at that time [4]. These electron microscopic changes were characterized by fragmentation of membranous organelles and scattered dark substances. It seems reasonable to consider that a biochemical mechanism responsible for slow neuronal damage in the hippocampus was already triggered at this stage following ischemia. Although we lack the knowledge of precise mechanisms underlying the delayed neuronal death, iron-dependent lipid peroxidation may be possibly involved at the time when ultrastructural alterations were initiated. At $48 \mathrm{~h}$ following ischemia, when morphological alterations in pyramidal cells became dramatically prominent, both lipid peroxide level and iron concentration in the damaged hippocampus were not significantly different from those of sham-operated group, suggesting that lipid peroxidation did not last up to $48 \mathrm{~h}$ following ischemia. It has been demonstrated that deferroxamine, a potent iron chelator, readily crosses the bloodbrain-barrier and is concentrated in brain tissues [22]. Treatment of animals with deferroxamine intraperitoneally $(50-300 \mathrm{mg} / \mathrm{kg}$ ) before and after ischemia for $20 \mathrm{~min}$, however, failed to diminish the morphological damage observed in the CA 1 subfield of the hippocampus following ischemia (data not shown). Antioxidants were not measured in the present study, therefore, ineffectiveness of deferroxamine on the progression of hippocampal death cannot necessarily exclude the possible involvement of free radical reactions. Further investigations concerning the metabolism of endogenous metal ions such as zinc following ischemia will be necessary.

The authors would like to thank Dr. K. Yagi, Institute of Applied Biochemistry, for his fruitful discussion and advise.

\section{REFERENCES}

1. Kirino, T. (1982): Delayed neuronal death in the gerbil hippocampus following ischemia. Brain Res., 239, 57-69.

2. Pulsinelli, W.A. (1985): Deafferentation of the hippocampus protects CA 1 pyramidal cells against ischemic injury. Stroke, 16, 144.

Vol. 3, No. 3, 1987 
3. Suzuki, R., Yamaguchi, T., Li, C.L., and Klazo, I. (1983): The effects of 5-minute ischemia in Mongolian gerbils. II. Changes of spontaneous neuronal activity in cerebral cortex and CA 1 sector of hippocampus. Acta Neuropathol. (Berl.), 60, 217-222.

4. Kirino, T., Tamura, A., and Sano, K. (1984): Delayed neuronal death in the rat hippocampus following transient forebrain ischemia. Acta Neuropathol. (Berl.), 64, 139-147.

5. Pulsinelli, W.A., Levy, D.E., and Duffy, T.E. (1982): Regional cerebral blood flow and glucose metabolism following transient forebrain ischemia. Ann. Neurol., 11, 499-509.

6. Pulsinelli, W.A., Duffy, T.E., Levy, D.E., Petito, C., and Plum, F. (1984): Ischemic injury to selectively vulnerable neurons in the rat, in Cerebral Ischemia, ICS 654, ed. by Bes, A., Braquet, P., Paoletti, R., and Siesjö, B.K., Excerpta Medica, Amsterdam, New York, Oxford, pp. 35-44.

7. Arai, H., Lust, W.D., and Passonneau, J.V. (1982): Delayed metabolic changes induced by 5 min of ischemia in gerbil brain. Trans. Am. Soc. Neurochem., 13, 177.

8. Siesjö, B.K. (1981): Cell damage in the brain: a speculative synthesis. J. Cereb. Blood Flow Metab., 1, 155-185.

9. Dienel, G.A. (1984): Regional accumulation of calcium in postischemic rat brain. J. Neurochem., 43, 913-925.

10. Kogure, K., Watson, B.D., Busto, R., and Abe, K. (1982): Potentiation of lipid peroxides by ischemia in rat brain. Neurochem. Res., 7, 437-454.

11. Yoshida, S., Inoh, S., Asano, T., Sano, K., Kubota, M., Shimazaki, H., and Ueta, N. (1980): Effect of transient ischemia on free fatty acids and phospholipids in the gerbil brain. $J$. Neurosurg., 53, 323-331.

12. Arai, H., Kogure, K., Sugioka, K., and Nakano, M. (1987): Importance of two iron-reducing systems in lipid peroxidation of rat brain: implications of oxygen toxicity in central nervous system. Biochem. Int., 14, 741-749.

13. Pulsinelli, W.A., and Brierley, J. (1979): A new model of bilateral hemispheric ischemia in the unanesthetized rat. Stroke, 10, 267-272.

14. Glowinski, J., and Iversen, L.L. (1966): Regional studies of catecholamine in the rat brain. I. The disposition of $\left[{ }^{3} \mathrm{H}\right]$ norepinephrine, $\left[{ }^{3} \mathrm{H}\right]$ dopamine and $\left[{ }^{3} \mathrm{H}\right]$ dopa in various regions of the brain. J. Neurochem., 13, 655-669.

15. Ohkawa, H., Ohishi, N., and Yagi, K. (1979): Assay for lipid peroxides in animal tissues by TBA reaction. Anal. Biochem., 95, 351-358.

16. Lowry, O.H., Rosebrough, N.J., Farr, A.L., and Randall, R.J. (1951): Protein measurement with the folin phenol reagent. J. Biol. Chem., 193, 265-275.

17. Ikeda, M., Watanabe, T., Hayashi, H., and Tsunoda, A. (1986): Toxicological evaluation of quartz and silica contents in studded tire-generated dust in the city of Sendai. Tohoku J. Exp. Med., 148, 207-211.

18. Hill, J.M., and Switzer, III, R.C. (1984): The regional distribution and cellular localization of iron in the rat brain. Neuroscience, 11, 595-603.

19. Francois, C., Nguyen-Legros, J., and Percheron, G. (1981): Topographical and cytological localization of iron in rat and monkey brains. Brain Res., 215, 317-322.

20. Halliwell, B., and Gutteridge, J.M.C. (1984): Oxygen toxicity, oxygen radicals, transition metals and disease. Biochem. J., 219, 1-14.

21. Zaleska, M.M., and Floyd, R.A. (1985): Regional lipid peroxidation in rat brain in vitro: possible role of endogenous iron. Neurochem. Res., 10, 397-410.

22. Keberle, H. (1964): The biochemistry of deferroxamine and its relation to iron metabolism. Ann. NY. Acad. Sci., 119, 758-768. 